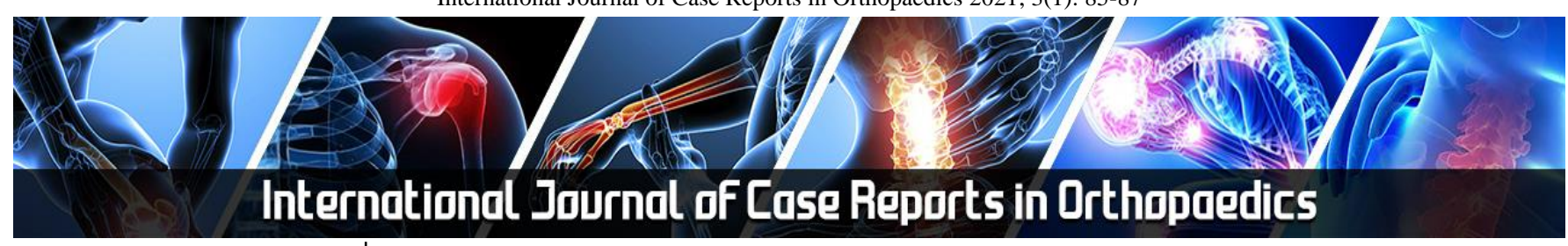

E-ISSN: 2707-8353 P-ISSN: 2707-8345 IJCRO 2021; 3(1): 85-87

Received: 22-01-2021 Accepted: 24-03-2021

\section{Ahsan Akhtar}

Post-CCT Upper Limb Fellow,

Wrightington Hospital,

Wigan, United Kingdom

Andrew Keightly

Consultant Shoulder and

Elbow Surgeon,

Royal Surrey NHS

Foundation Trust, Wigan,

United Kingdom

\section{Puneet Monga}

Consultant Shoulder Surgeon,

Wrightington Hospital,

Wigan, United Kingdom
Corresponding Author: Ahsan Akhtar

Post-CCT Upper Limb Fellow,

Wrightington Hospital,

Wigan, United Kingdom

\section{The use of an anti-rotation wire in custom-made shoulder arthroplasty}

\author{
Ahsan Akhtar, Andrew Keightly and Puneet Monga
}

DOI: https://doi.org/10.22271/27078345.2021.v3.i1b.55

\begin{abstract}
Shoulder arthroplasty have become increasingly more common in the UK and has been shown to be a successful operation that can significantly improve quality of life. Advanced arthritic patterns are frequently being identified with expansive bone loss which cannot be managed via traditional arthroplasty techniques. As no reliable landmarks are available intra-operatively to determine glenoid morphology and scapular plane, the use of patient-specific glenoid guides are becoming more commonplace in order to prepare the glenoid and accurately guide the placement of the glenoid component during conventional and reverse shoulder arthroplasty. A single, central guidewire that is used to orientate the custom base-plate permits a degree of rotational malalignment around its central axis. We describe the use of an anti-rotation guidewire to aid in implantation of a definitive custommade glenoid base-plate, which is safe, effective and reproducible.
\end{abstract}

Keywords: guidewire, custom-made, shoulder arthroplasty

\section{Introduction}

Shoulder arthroplasty have become increasingly more common in the UK ${ }^{[1]}$ and has been shown to be a successful operation that can significantly improve quality of life. [2, 3] Advanced arthritic patterns are frequently being identified with expansive bone loss ${ }^{[4,5]}$ often encountered during revision arthroplasty - which cannot be managed via traditional arthroplasty techniques.

Custom-made implants utilise CT scans to map severe glenoid bone loss to design a patient specific prosthesis ${ }^{[6]}$, in order to ensure precise positioning of the glenoid component, which is crucial to avoid early loosening.

In this technical note, we aim to describe the use of an anti-rotation guidewire to aid in implantation of a definitive custom-made glenoid base-plate. This technique is safe, effective and reproducible.

\section{Technique}

The Promade system (Lima Corporate, Udine, Italy) allows a custom-made prosthesis to overcome significant bone loss and restore optimum shoulder mechanics. The current design utilises a central guide wire with a custom jig to guide glenoid component positioning (fig 1).

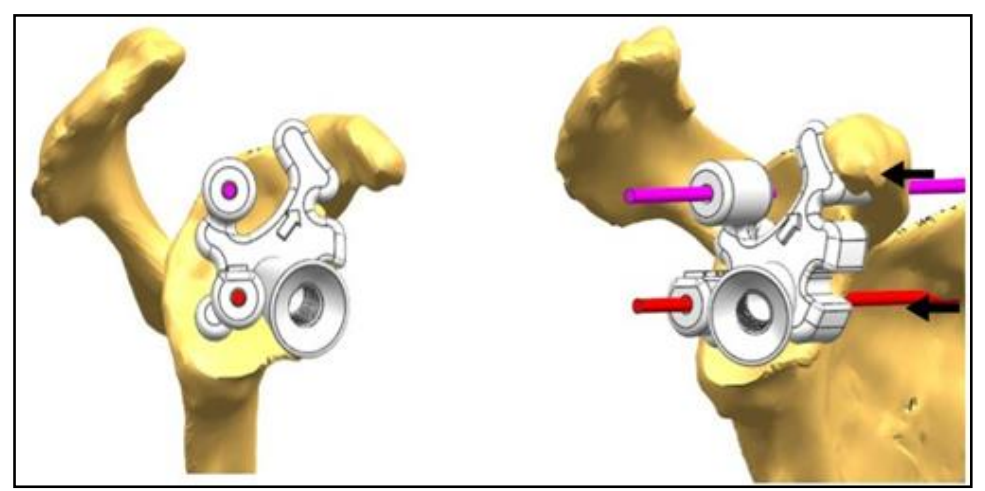

Fig 1: AP and lateral views of a custom glenoid jig demonstrating the placement of a central guidewire (red) and anti-rotation wire (purple). 
Although such implants correspond accurately to the glenoid defect, a lack of rotational control using a single, central guidewire can potentially lead to imprecise seating of the custom base- plate onto the native glenoid.

The addition of a second, anti-rotation wire into the design of custom-made glenoid jigs can maximise the accuracy of glenoid placement; such a wire is placed superiorly through the same custom jig. The position of this wire then corresponds to a matched groove designed on the superior surface of the definitive glenoid base-plate to ensure accurate rotational implantation of the custom implant (fig 2).

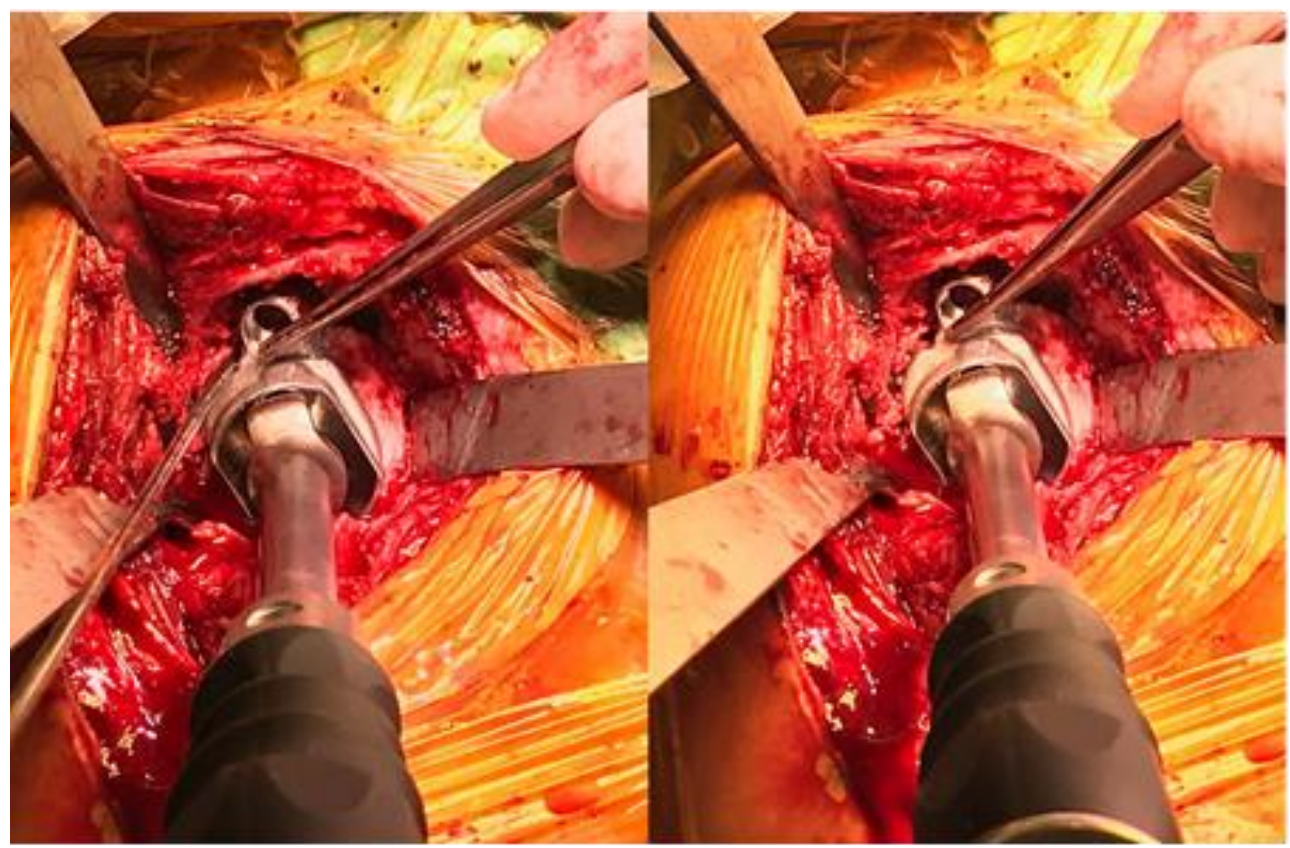

Fig 2: Insertion of the definitive glenoid base plate demonstrating the anti-rotation wire and groove in which this sits on the definitive implant

\section{Discussion}

As no reliable landmarks are available intra-operatively to determine glenoid morphology and scapular plane, the use of patient-specific glenoid guides are becoming more commonplace in order to prepare the glenoid and accurately guide the placement of the glenoid component during conventional and reverse shoulder arthroplasty ${ }^{[7,8]}$. The use of a proposed second wire, which stabilizes and prevents inadvertent component rotation, provides an element of control considering that a mean deviation of approximately $5-10^{\circ}$ for both version and inclination has previously been found when glenoid components are positioned with standard instrumentation ${ }^{[9,10]}$.

Custom-made implants rely on precise implantation of the prosthesis. Optimal positioning of the single, central guidewire requires posterior retraction of the humerus and deltoid, with adequate exposure of the glenoid, as well as removal of all soft tissues, being important steps in allowing stable positioning of the custom-made glenoid jig. A single, central guidewire that is used to orientate the custom baseplate permits a degree of rotational malalignment around its central axis. The authors believe the addition of an antirotation wire to the planning and design of the custom prosthesis adds a further degree of accuracy to the system without adding constraints to surgical technique or compromising osseousor base-plate stability.

\section{References}

1. National Joint Registry for England, Wales and Northern Ireland; 16th Annual Report 2019. https://reports.njrcentre.org.uk/Portals/0/PDFdownloads /NJR\%2016th\%20Annual\%20Report\%202019.pdf.
2. Cofield RH. Total shoulder arthroplasty with the Neer prosthesis. J Bone Joint Surg Am 1984;66(6):899-906. doi: 10.2106/00004623-198466060-00010.

3. Khan A, Bunker TD, Kitson JB. Clinical and radiological follow-up of the Aequalis third-generation cemented total shoulder replacement: a minimum tenyear study. J Bone Joint Surg Br 2009;91(12):1594600. doi: 10.1302/0301-620X.91B12.22139.

4. Malhas A, Rashid A, Copas D, Bale S, Trail I. Glenoid bone loss in primary and revision shoulder arthroplasty. Shoulder Elbow. 2016;8(4):229-40. doi: $10.1177 / 1758573216648601$.

5. Boileau P. Complications and revision of reverse total shoulder arthroplasty. Orthop Traumatol Surg Res. 2016;102(1Suppl):S33-43. doi: 10.1016/j.otsr.2015.06.031

6. Elliott R, Dallalana R. Patient-specific Instrumentassisted Structural Glenoid Bone Grafting in Reverse Shoulder Arthroplasty. Techniques in Shoulder \& Elbow Surgery 2017;18(4):145-150. doi: 10.1097/BTE.00000000000001

7. Suero EM, Citak M, Lo D, Krych AJ, Craig EV, Pearle AD. Use of a custom alignment guide to improve glenoid component position in total shoulder arthroplasty. Knee Surg Sports Traumatol Arthrosc. 2013;21(12):2860-6. doi: 10.1007/s00167-012-2177-

8. Gauci M, Boileau P, Baba M, Chaoui J, Walch G. Patient-specific glenoid guides provide accuracy and reproducibility in total shoulder arthroplasty, Bone Joint J 2016;98-B:1080-5. doi: 10.1302/0301620X.98B8.37257.

9. Nguyen D, Ferreira LM, Brownhill JR, King GJ, Drosdowech DS, Faber KJ et al. Improved accuracy of 
computer assisted glenoid implantation in total shoulder arthroplasty: an in-vitro randomized controlled trial. J Shoulder Elbow Surg. 2009;18(6):907-14.

doi: 10.1016/j.jse 2009.

10. Verborgt O, De Smedt T, Vanhees M, Clockaerts S, Parizel PM, Van Glabbeek F. Accuracy of placement of the glenoid component in reversed shoulder arthroplasty with and without navigation. J Shoulder Elbow Surg. 2011;20(1):21-6.

doi: $10.1016 /$ j.jse.2010.07.014 